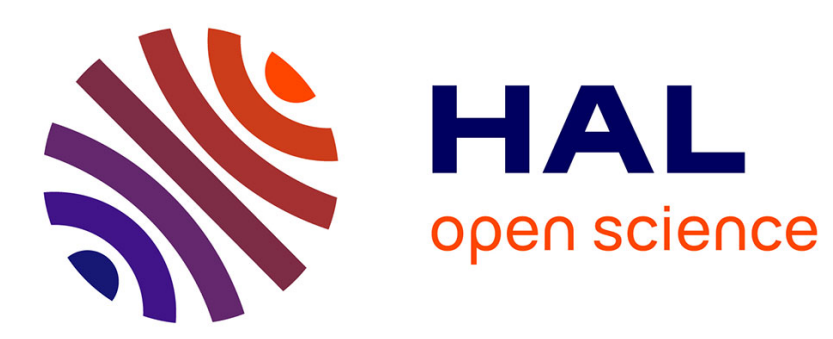

\title{
Origin of dynamical instabilities in some simulated 2D Materials: GaSe as a case study
}

Silvana Radescu, Denis Machon, Patrice Mélinon

\section{To cite this version:}

Silvana Radescu, Denis Machon, Patrice Mélinon. Origin of dynamical instabilities in some simulated 2D Materials: GaSe as a case study. Physical Review Materials, 2019, 3 (7), pp.074002. 10.1103/PhysRevMaterials.3.074002 . hal-02991182

\section{HAL Id: hal-02991182 \\ https://hal.science/hal-02991182}

Submitted on 5 Nov 2020

HAL is a multi-disciplinary open access archive for the deposit and dissemination of scientific research documents, whether they are published or not. The documents may come from teaching and research institutions in France or abroad, or from public or private research centers.
L'archive ouverte pluridisciplinaire HAL, est destinée au dépôt et à la diffusion de documents scientifiques de niveau recherche, publiés ou non, émanant des établissements d'enseignement et de recherche français ou étrangers, des laboratoires publics ou privés. 


\title{
Origin of dynamical instabilities in some simulated 2D Materials: \\ GaSe as a case study
}

\author{
Silvana Radescu ${ }^{1}$, Denis Machon ${ }^{2,3}$, Patrice Mélinon ${ }^{2}$ \\ 1 - Departamento de Física and Instituto Universitario de Materiales y \\ Nanotecnología, Universidad de La Laguna, La Laguna 38200, Tenerife, Spain \\ 2 - Institut Lumière Matière, Université de Lyon, Université Claude Bernard Lyon 1, \\ CNRS UMR 5306, 69622 Villeurbanne, France \\ 3 - Laboratoire Nanotechnologies et Nanosystèmes (LN2), CNRS UMI-3463, \\ Université de Sherbrooke, Institut Interdisciplinaire d'Innovation Technologique \\ (3IT), Sherbrooke, Québec, Canada
}

\begin{abstract}
Following the emergence of 2D materials, a huge amount of work has been dedicated to this new class of materials. Numerical simulations have proven to be powerful to analyze and predict structure-properties relationships. However, a recurrent issue that arises in some 2D compounds is the appearance of a dynamical instability as an unstable phonon branch in a small pocket close the Brillouin zone center. The origin (numerical and/or physical) of this instability is hardly discussed. Here, using a rising 2D material, GaSe, as a case study, this issue is tackled by discussing the numerical techniques that may be used to lift the instability but also by understanding the fundamental origin of it. The interlayer distance is the crucial parameter and the ionicity of the compounds is the key physical property governing the propensity for instability. In many works, this distance is arbitrarily fixed to a value for which the absence of interactions between the periodic images of the layers is assumed. A careful control of the effect of this distance on the stability is required prior to subsequent calculations of physical properties.
\end{abstract}




\section{Introduction}

The isolation of a single graphene layer by mechanical exfoliation in 2004 has generated a huge research activity dedicated to 2D materials and, consequently, renewed the interest for lamellar compounds $[1,2,3]$. These materials exhibit tight bounds in the plane and weak van der Waals interlayer bounds. They are likely to give a single layer by exfoliation. In the last few years, a wide variety of $2 \mathrm{D}$ compounds has been synthesized. Their electronic properties are different from those of bulk material and can vary from wide bandgap semiconductor to semi-metal. For instance, whilst the bulk material is an indirect semiconductor, $\mathrm{MoS}_{2}$ single layer becomes a direct semiconductor and gives rise to a photoluminescence signal $[4,5]$.

Experiments on 2D materials are demanding and a screening step using simulations to identify potentially interesting $2 \mathrm{D}$ samples is crucial. For instance, to investigate the electronic properties of $2 \mathrm{D}$ materials, ab initio simulations are powerful techniques. However, little attention is dedicated to the stability of the 2D material i.e., on the calculations of the phonon band structure in addition to the electronic one, even though it has been shown that this aspect is important as it reflects the stability conditions of the nanostructure [6]. Actually, even if the total energy of a structure can be minimized, its stability cannot be assured as long as the dynamical stability (absence of unstable phonon) is not confirmed.

In the recent literature, a redundant point has appeared in simulations of $2 \mathrm{D}$ materials. When calculating the phonon dispersion curves, unstable phonon branch in a small pocket close the Brillouin zone center was found in few cases [7, 8]. This was interpreted as a non-physical effect related to the difficulty in achieving numerical convergence for the flexural phonon mode in 2D materials $[9,10]$.

However, several questions remain. First, what is the numerical origin of this effect? Then, why only some compounds are affected and not all of them? Finally, are we sure that no physical underlying reason may be invoked?

To tackle these issues, we propose to study a rising $2 \mathrm{D}$ materials, GaSe $[11,12,13$, $14,15,16,17]$ and to investigate the dynamical instability by using different numerical techniques. In this work, we will show the importance of determining the 
appropriate supercell during ab initio simulations to determine the physicals properties such as the phonon dispersion curves and, consequently, the dynamical stability.

The paper is divided as follow: in the next section, the 3D and 2D structures of GaSe will be introduced. Then, the computational details will be given. In section IV, simulations of 3D GaSe will be presented with an emphasis on the stability of the structure. This section continued with the same study but on 2D GaSe.

\section{Tri- and bi-dimensional GaSe structures}

Under normal conditions, bulk GaSe may adopt one of the four known crystal polytypic structures labelled $\beta, \gamma, \delta$, and $\varepsilon$ [18]. Despite efforts to synthesize new polymorphs of this material, no other crystalline metastable phase at normal conditions has been artificially produced in laboratories.

These polytypic phases consist of a crystalline layered arrangement in which each atom of $\mathrm{Ga}$ is surrounded by three $\mathrm{Se}$ atoms and one $\mathrm{Ga}$ atom. The structure of all polytypes is very similar, with covalently bonded hexagonal slabs of $\mathrm{Ga}$ and Se atoms arranged according to a certain stacking sequence. The adjacent slabs of layers are held together by van der Waals interactions which act along the stacking axis (c-axis). The most common polytype is the $\varepsilon$-type (Space group $\mathrm{N}^{\circ} 187-P \overline{6} m 2$ ) (Figure 1).

From the 3D material, quasi-2D sheets consisting of slabs containing a few layers may be obtained by mechanical exfoliation [19]. The structure of a single monolayer may be generated using the structure of the $\varepsilon$ polymorph. Layers are isolated and have a point group symmetry $\mathrm{D}_{3 \mathrm{~h}}$. Contrary to Transition Metal Dichalcogenides (TMD), there is no change of the point group symmetry when going from the bulk to the single layer as there is not an inversion center linking two adjacent layers. Starting from another polymorph would lead to same monolayer structure as the only difference between the different polymorphs is the stacking sequence of the layers. Therefore, the simulation supercell only consists in a larger c-axis parameter (Figure 1).

The monolayer structure is then numerically generated by the isolation of a layer from the bulk materials as it would be in the case of a mechanical exfoliation. It worth noting that, to the best of our knowledge, there is no alternative atomic configuration for the monolayer. 


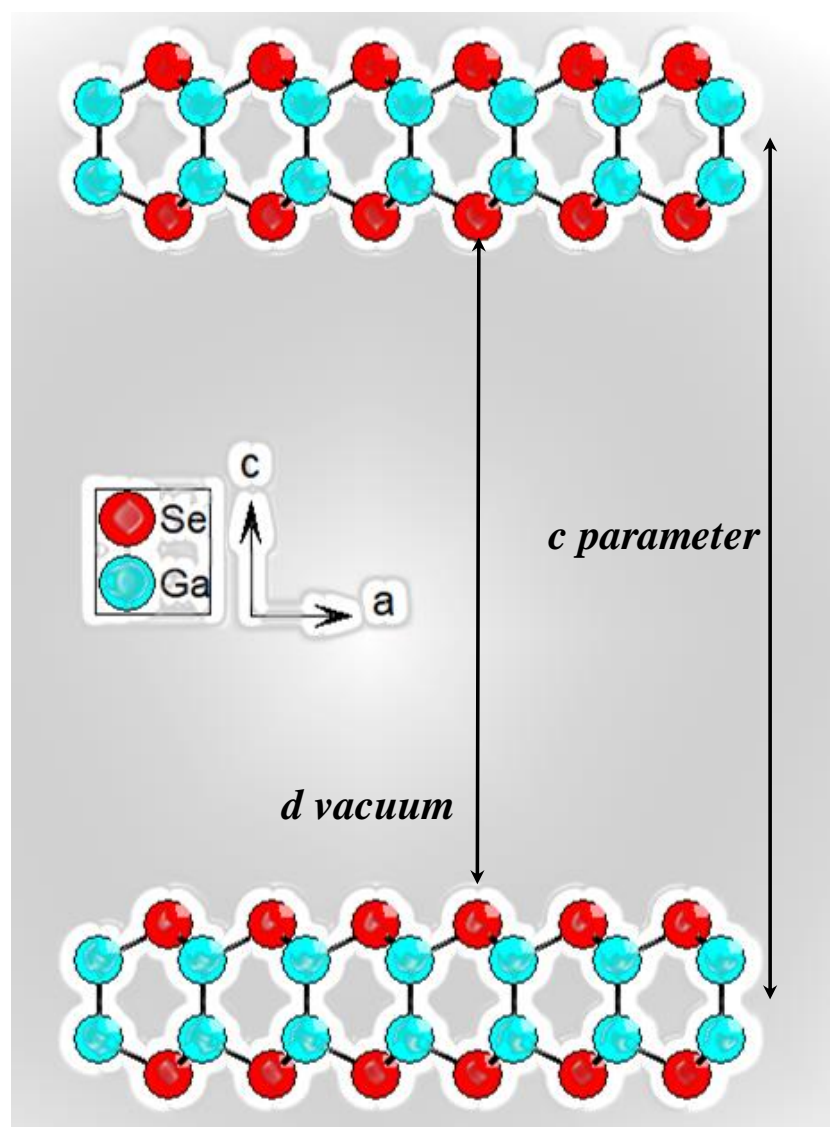

Figure 1 - Simulation configuration showing the required periodicity along the c-axis. The difference between $2 \mathrm{D}$ and $3 \mathrm{D}$ structures is only related to the value of the cparameter and, consequently, to the distance between adjacent layers referred as $d$ vaccum.

\section{Computational details}

All the calculations were done within the first-principles theoretical framework of the density functional theory (DFT) implemented in the computational scheme of pseudopotentials and plane waves in the projector-augmented wave scheme. The Vienna Ab Initio Simulation Package (VASP) code was employed [20, 21 ]. We did calculations with the Perdew-Burke-Ernzerhof (PBE) version of the generalized gradient approximation (GGA) to the exchange-correlation (XC) functionals [ 22]. The van der Waals (vdW) corrections to the total energy were taken into account within the DFT using Grimme's so-called D2 method [23].

The $4 \mathrm{~s}$ and $4 \mathrm{p}$ outermost electrons of $\mathrm{Ga}$ and Se were dealt with explicitly in the valence, the rest of the electrons being considered frozen at the core. The cutoff in the kinetic energy of the plane-waves basis set was $310 \mathrm{eV}$ which together with the use of 
a dense $15 \times 15 \times 5$ Monkhorst-Pack grid of k-points for integrations in reciprocal space ensured a convergence in the energy differences between phases of around $1 \mathrm{meV}$. All the results show here correspond to zero temperature, with zero-point motion of lattice vibrations neglected.

To test the reliability of our simulations, we first investigate the stability of bulk $\varepsilon$ GaSe. A full structural optimization of both the internal parameters and the cell shape was performed through the calculation of the forces on atoms and the components of the stress tensor. The structural relaxation was deemed complete when the atomic forces were less than $0.005 \mathrm{eV} . \AA^{-1}$ and the anisotropy in the diagonal components of the stress tensor was less than $0.1 \mathrm{GPa}$.

For the monolayer of GaSe simulated within the periodically repeated scheme imposed by the technique of calculation, a set of $15 \times 15 \times 1 \mathrm{k}$-points grid was used. A vacuum region between monolayers is set to avoid interaction between the periodic images of the layers (Figure 1). To obtain the unstrained configuration, the atomic positions and lattice vectors were fully relaxed and the optimized (relaxed) coordinates were then used for the self-consistent cycle.

The electron localization function (ELF) was calculated from the valence electronic density obtained within the pseudopotential scheme implemented in VASP (where the core electrons are considered frozen and not explicitly considered). The visualization of the ELF isosurface was done with VESTA 3.4.3.

To provide a rigorous analysis of the stability in $2 \mathrm{D} \mathrm{GaSe}$ we have considered a very high accuracy in the calculation of the forces. A structure is stable only if the calculated frequencies of all its phonon modes in the BZ are positive; otherwise, the presence of imaginary frequencies indicates an instability.

In order to calculate the phonons at the zone center as well as in the whole Brillouin zone we used both the small-displacements method (also called sometimes the direct, supercell or force constant method) as implemented in the PHON code [24] and the density functional perturbation theory (DFPT, or linear-response method) as implemented in the VASP code, using the Phonopy package to calculate the phonon density of state $[25,26]$. Both methods hinge at the core on the linear relationship 
between the forces on the atoms originated by an atomic displacement from equilibrium and the displacement itself.

In the small-displacements method, one considers an appropriate supercell corresponding to the equilibrium configuration and displaces one atom at a time, calculating the resulting forces on all the supercell sites. The required number of independent displacements depends on the symmetry of the independent sites in the crystal. From the restoring forces for the set of independent displacements one can then approximate the force-constants matrix and, from that, construct the dynamical matrix, whose eigenvalues are the squares of the phonon frequencies and the corresponding eigenvectors are the polarization vectors of the modes. This method does not require any a priori information about the eigenvectors, but requires the force calculation, needs a supercell to extract properties for non-zero wavevector phonons and is very expensive numerically for long ranged interactions.

Within DFPT, one achieves the calculation of the phonons using perturbative techniques within linear response theory as applied specifically to the density functional formalism in periodic solids. The big advantage of DFPT versus the smalldisplacements method is that it works directly in the reciprocal space, so it gives directly the dynamical matrix (obtained from the second derivatives of the total energy within standard perturbation schemes) for any arbitrary phonon wavevector with no need to use a supercell.

\section{III.Results and discussion}

\section{Stability of 3D bulk GaSe}

As GaSe is a lamellar compound, interslab van der Waals forces may play a significant role in the structural stability as shown in Figure 2. From preliminary test calculations made using different pseudopotentials and exchange-correlation functionals it turned out that the best results were provided by the GGA-PBEvdw functional. In the energy $v s$ volume curves shown in this figure, a well defined minimum in the E-V curve can be obtained i.e., a stable structure under normal conditions, only by including explicitly the van der Waals interactions. On the contrary, if the vdW interactions are not explicitly introduced, no defined minima indicating a stable structure may be observed. A defined structure is obtained under a 
slight compressive stress according to the calculated P-V curves with and without vdW corrections [27].

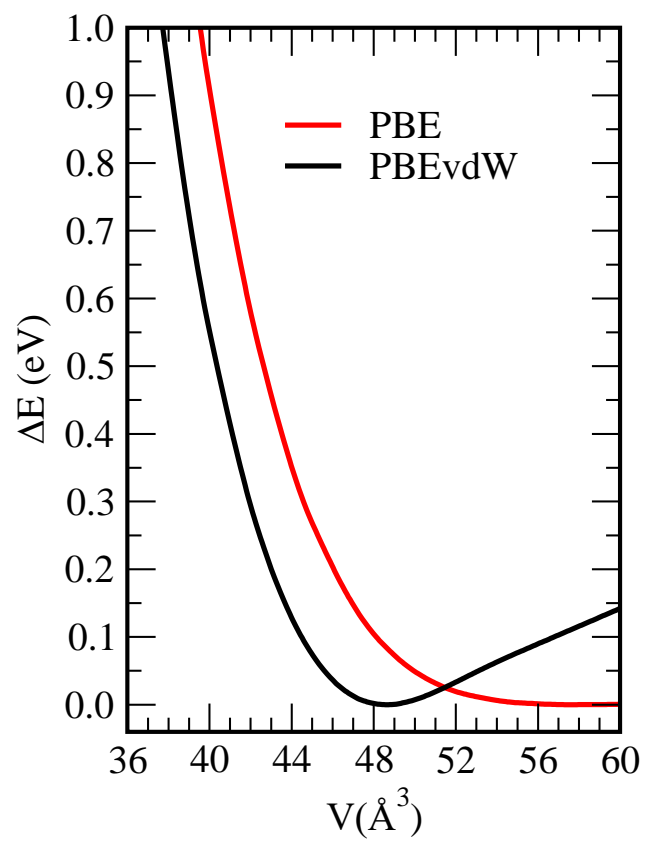

Figure 2. Energy-Volume curves (per formula unit) for the $\varepsilon$-polytype of GaSe, with $(\mathrm{PBEvdW})$ and without (PBE) van der Waals interactions.

It is worth noting that including the van der Waals interactions (PBEvdW) induces a volume reduction of almost $10 \%$ compared to the situation without these interactions $(\mathrm{PBE})$. This is an indication of the importance of the inter-plan interactions as the intra-plan interactions are not strongly affected by the introduction of the out-of-plane interactions (Table I). The calculations of the structure 3D-GaSe using vdW are in very good agreement with the cell parameters and atomic positions observed experimentally (Table I). 
Table I - Crystallographic description of equilibrium bulk GaSe with (PBEvdW) and without (PBE) van der Waals corrections. The value of the volume $\mathrm{V}_{\mathrm{o}}$ for the PBE case corresponds to a slightly compressed state that ensures a defined structure [27].

\begin{tabular}{|c|c|c|}
\hline 3D-\&-GaSe (Bulk) & Space group & Atomic position \\
\hline $\begin{array}{c}\mathrm{V}_{\mathrm{o}}=48.28 \AA^{3} \\
\text { Experimental data } \\
{[18,28]}\end{array}$ & $\begin{array}{c}\mathrm{N}^{\circ} 187-P \overline{6} m 2 \\
\mathrm{a}=\mathrm{b}=3.74 \AA \text { 的 }=15.92 \AA \\
\text { alpha }=\text { gamma }=90.00 \\
\text { beta }=120.00\end{array}$ & $\begin{array}{c}\text { Ga } 2 \mathrm{~g}(0.0,0.0,0.075) \\
\mathrm{Ga} 2 \mathrm{i}(2 / 3,1 / 3,0.57) \\
\mathrm{Se} 2 \mathrm{i}(2 / 3,1 / 3,0.85) \\
\mathrm{Se} 2 \mathrm{~h}(1 / 3,2 / 3,0.65)\end{array}$ \\
\hline $\begin{array}{c}\mathrm{V}_{\mathrm{o}}=54.4 \AA^{3} \\
\text { Present work } \\
\text { XC: PBE }\end{array}$ & $\begin{array}{c}\mathrm{N}^{\circ} 187-P \overline{6} m 2 \\
\mathrm{a}=\mathrm{b}=3.82 \AA ; \mathrm{c}=18.42 \AA \\
\text { alpha }=\text { gamma }=90.00 \\
\text { beta }=120.00\end{array}$ & $\begin{array}{ll}\mathrm{Ga} & 2 \mathrm{~g}(0.0,0.0,0.067) \\
\mathrm{Ga} & 2 \mathrm{i}(2 / 3,1 / 3,0.567) \\
\mathrm{Se} & 2 \mathrm{i}(2 / 3,1 / 3,0.869) \\
\mathrm{Se} & 2 \mathrm{~h}(1 / 3,2 / 3,0.631)\end{array}$ \\
\hline $\begin{array}{l}\mathrm{V}_{\mathrm{o}}=48.68 \AA^{3} \\
\text { Present work } \\
\mathrm{XC}: \text { PBEvdW }\end{array}$ & $\begin{array}{c}\mathrm{N}^{\circ} 187-P \overline{6} m 2 \\
\mathrm{a}=\mathrm{b}=3.75 \AA ; \mathrm{c}=15.93 \AA \\
\text { alpha }=\text { gamma }=90.00 \\
\text { beta }=120.00\end{array}$ & $\begin{array}{ll}\mathrm{Ga} & 2 \mathrm{~g}(0.0,0.0,0.076) \\
\mathrm{Ga} & 2 \mathrm{i}(2 / 3,1 / 3,0.576) \\
\mathrm{Se} & 2 \mathrm{i}(2 / 3,1 / 3,0.849) \\
\mathrm{Se} & 2 \mathrm{~h}(1 / 3,2 / 3,0.651)\end{array}$ \\
\hline
\end{tabular}

Next, phonon dispersion curve of $\varepsilon$-GaSe structure obtained using the PBEvdW formalism has been calculated using the small-displacement method (Figure 3). No imaginary phonons frequencies are obtained which indicates dynamical stability. Some phonon frequencies at the zone Brillouin center can be compared to Raman shift measured experimentally and are in good agreement [29].

In conclusion, concerning 3D $\varepsilon-\mathrm{GaSe}$, structural stability is only obtained when including explicitly van der Waals (vdW) interactions. Not taking them into account produces energy-volume curves without a clear minimum (equilibrium state at zero pressure), which relates to the stabilizing role of the vdW interactions. In addition, calculations of the vibrational dispersion curves using the finite displacement method do not show any dynamical instability of the structure. 


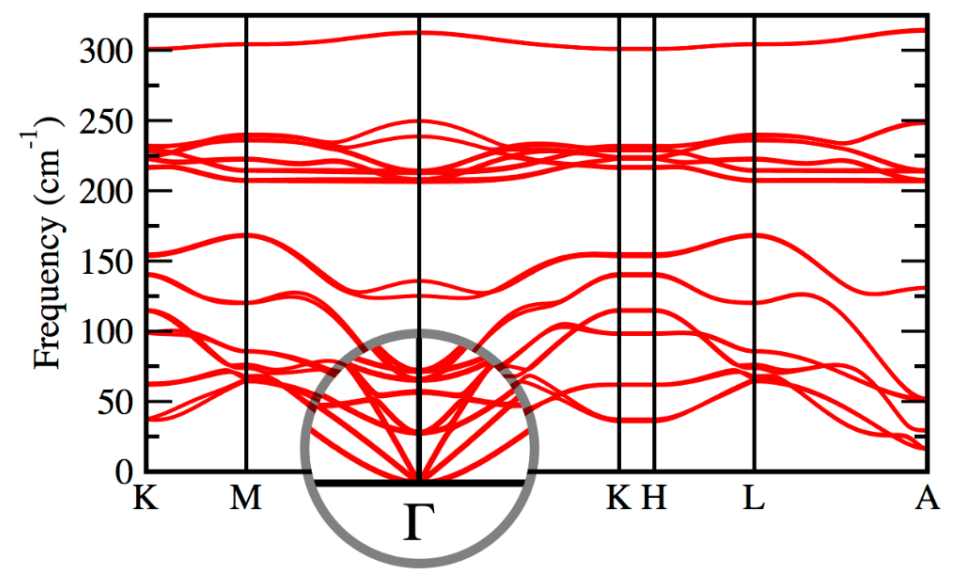

Figure 3. Phonon dispersion curves of bulk $\varepsilon$-GaSe using the small-displacements method

\section{Generation and Stability of $2 \mathrm{D}$ GaSe}

Because of the periodicity required in the simulation cell in all directions, there is a stacking sequence along the axis perpendicular to the layers (c-axis) similar to the 3D case (Figure 1). It is necessary to choose a sufficiently large distance between two adjacent layers to consider them as if they were isolated without out-of-plane interactions. However, increasing the cell volume induces an increased computation time. Therefore, the choice of the c-parameter length must be a compromise. Usually, it is admitted that an increase by $50 \%$ of the c-axis is sufficient to consider the layers as isolated. For instance, Ataca et al. in a study comparing 88 different $\mathrm{MX}_{2}$ compounds fixed a value of $15 \AA$ as interslab distance for all of them [7].

In a first test (monolayer 1 in Table II), we fixed a c-axis value of $24.29 \AA$ which corresponds approximately a 50\% expansion of the interlayer distance. This corresponds to vacuum with a width $\sim 19.5 \AA$ (distance between two nearest Se atoms belonging to different monolayers) intercalated between slabs along the stacking direction (Figure 1). 
Table II - crystallographic description of unstrained configuration of the $2 \mathrm{D} \mathrm{GaSe}$

\begin{tabular}{c|c|c}
\hline \hline \multirow{2}{*}{ 2D-GaSe (XC: PBE) } & Space group & Atomic position \\
\hline Monolayer 1 & $\begin{array}{c}\mathrm{N}^{\circ} 187-P \overline{6} m 2 \\
\mathrm{a}=\mathrm{b}=3.82 \AA ; \mathrm{c}=24.29 \AA \\
\mathrm{alpha}=\mathrm{gamma}=90.00 \\
\text { beta=120.00 }\end{array}$ & $\begin{array}{c}\mathrm{Ga} 2 \mathrm{~g}(0.0,0.0,0.551) \\
\mathrm{Se} 2 \mathrm{i}(2 / 3,1 / 3,0.401)\end{array}$ \\
\hline Monolayer 2 & $\begin{array}{c}\mathrm{N}^{\circ} 187-P \overline{6} m 2 \\
\mathrm{~b}=3.82 \AA ; \mathrm{c}=27.64 \AA \\
\mathrm{alpha}=\mathrm{gamma}=90.00 \\
\text { beta }=120.00\end{array}$ & $\begin{array}{c}\mathrm{Ga} 2 \mathrm{~g}(0.0,0.0,0.545) \\
\mathrm{Se} 2 \mathrm{i}(2 / 3,1 / 3,0.413)\end{array}$ \\
\hline \hline
\end{tabular}

The structure has been simulated by using the PBE functional i.e., without introducing the vdW corrections. Phonon dispersion curves for the GaSe monolayer have been calculated using the small-displacement method (Figure 4a) to evaluate the dynamical stability. As reported for several 2D materials, instability occurring at longwavelength acoustical modes is observed with the appearance of unstable modes (imaginary frequencies) at the center of the Brillouin zone around the $\Gamma$ point.

Such situation is encountered for several compounds without a clear explanation of the reasons leading to a dynamical instability in some cases but not in others. Our working hypothesis is that the reason is beyond numerical methodology but rather reflects some physical phenomenon. 
(a)

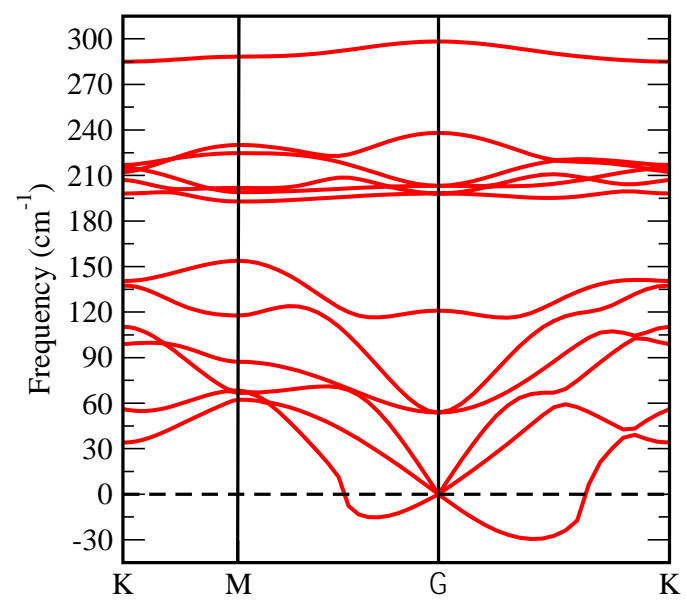

(b)

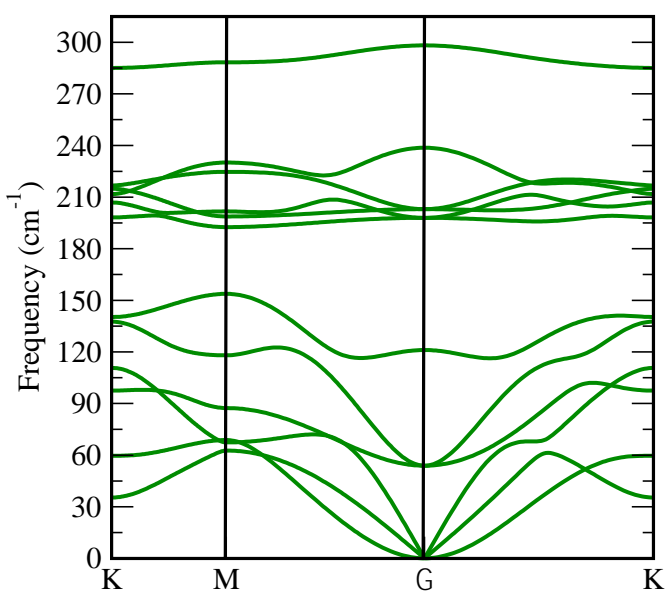

Figure 4 - Phonon dispersion curves of $2 \mathrm{D}$ GaSe with a c-parameter value of $24.3 \AA$ (monolayer 1 in Table II) calculated by (a) the small-displacements method (b) the density-functional perturbation theory (DFPT) method.

A first possibility would be that the simulated structure should undergo a structural phase transition to a more stable structure. This is the case in some TMD 2D materials where two phases ( $\mathrm{H}$ and $\mathrm{T}^{\prime}$ ) may be observed [30, 31, 32, 33]. However, concerning $2 \mathrm{D} \mathrm{GaSe}$, there is no experimental report in the literature of any structural distortion.

A different numerical technique to simulate the phonon dispersion curve is the density functional perturbation theory (DFPT). Surprisingly, the dynamical instability is not observed anymore (Figure 4b). The different conclusions from both methodologies certainly lie in the fact that DFPT is an approach taking into account small perturbations from a ground-state system. It has been mentioned that coulombic interactions between layers may exist in GaSe following reflectivity and Raman measurements $[18,34]$, we assume that some out-of-plane interactions remains despite the spacing of $\sim 20 \AA$ between the adjacent monolayer in the simulation supercell.

Therefore, a new set of calculations has been performed with a c-parameter value multiplied by a factor of 3 i.e., with a c-parameter equal to $48.7 \AA$, corresponding to a Se-Se distance of $\sim 39 \AA$. In that case, using the small displacement method, the dynamical instability disappears totally, leading to a stable structure (Figure 5). This result clearly indicates that the origin of the instability is dominated by the interplanar interactions. 


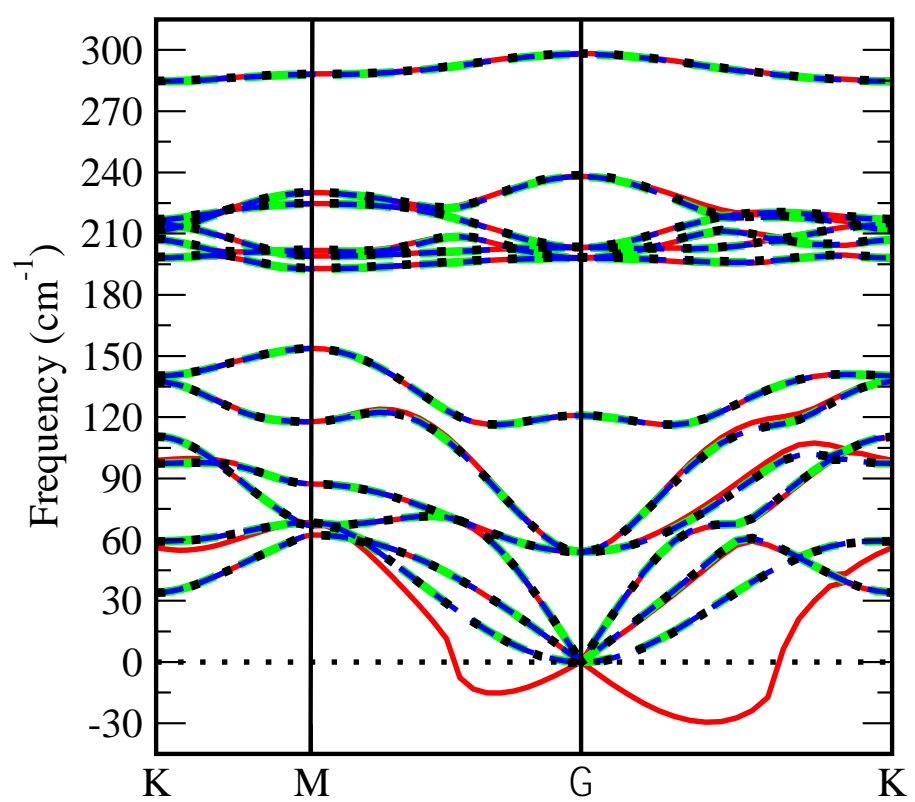

Figure 5 - Comparison of the phononic (vibrational) band structure calculated by the small displacements method for the value of the c parameter $24.3 \AA$ (red line) (monolayer 1 in Table II), $27 \AA$ (blue dot line) (monolayer 2 in Table II) and $48.7 \AA$ (black line). Green line corresponds to the results using DFPT calculation with $\mathrm{c}=$ $24.3 \AA$.

However, with the increase of the c-parameter, the computational time increases significantly, and it is mandatory to find the appropriate value for this parameter. In the search for a minimal c-parameter value that leads to a stable structure, it has been found that a value of the c-axis length of $\sim 27 \AA$ is necessary to lift the dynamical instability when using the small-displacement formalism (Figure 5). Surprisingly, this optimum between the cell parameter and the dynamical stabilization is 1.5 times the value of the c-axis obtained when the 3D-GaSe has been simulated without the introduction of van der Waals interactions i.e, that does not have energy minimum (Figure 1 and PBE in Table I). This result is central as it contradicts a standard procedure that consists in optimizing the equilibrium configuration of the $3 \mathrm{D}$ structure and, then, to use these results to define a 2D structure. Paradoxically, the 2D structure should be optimized starting from a 3D bulk structure simulated without vdW corrections.

Our results are in line with the emerging field of remote epitaxy [35]. This technique consists in inserting graphene layers between the substrate and the grown material. An 
epitaxial growth was observed despite the presence of these graphene layers indicating a long-rage interaction between materials. The potential field from ionic-bonded materials such as $\mathrm{GaN}$ or GaAs is strong enough to penetrate through few-layers of graphene. In addition, depending on the polarity of the materials (substrates and 2D), the interactions can be modulated [36]. For instance, GaN can grow epitaxially through two graphene layers but GaAs, which is less ionic, can only grow epitaxially on one graphene layer.

This experimental evidence is correlated with our results that indicate the existence of long-range interactions that may influence the simulation of an insufficiently isolated monolayer.

GaSe has an intermediate ionic character with an ionicity $f=0.56$ [37] that may be sufficient to induce long-range interactions on distance as long as $\sim 2 \mathrm{~nm}$. The analysis of the electron localization function (ELF) yields a topological account of the distribution and localization of these valence electrons and provides useful information on the (ionic) features of the studied material. In Figure 6, we show ELF plots for the GaSe monolayer in two different projections and a larger region of localization around the $\mathrm{Se}$ atoms is clearly observed. The domain of localization between the Ga-Se-Ga segments are clearly distinguishable from the plots of the ELF profiles shown in Figure 6b. There is a moderately higher value for the ELF in the region of space corresponding to the Ga-Se bonds (with a value close to 0.88 ) and lower values of ELF (close to 0.4) for Se atoms. Lower values of ELF level are usually employed to describe ionic systems. Therefore, the results of the ELF analysis support the ionic character of GaSe.

When substituting Se by Te that reinforces the ionic character in $\varepsilon-G_{a S e} e_{1-x} T e_{x}$, the interlayer bonding energy increases drastically [38] indicating a relationships between interlayer interaction and polarity. In the same vein, simulations of 2D InSe show dynamical instability and its ionicity is higher than $\mathrm{GaSe}(\mathrm{f}=0.64$ [37]).

On the other side, $\mathrm{MoS}_{2}$ is a typical example of 2D TMDs. Despite a substantial amount of numerical studies of this compound, no dynamical instability has been reported. This may be explained by the fact that its ionicity is much lower than $\mathrm{GaSe}, \mathrm{f}$ $=0.12-0.13[39,40]$. 
(a)

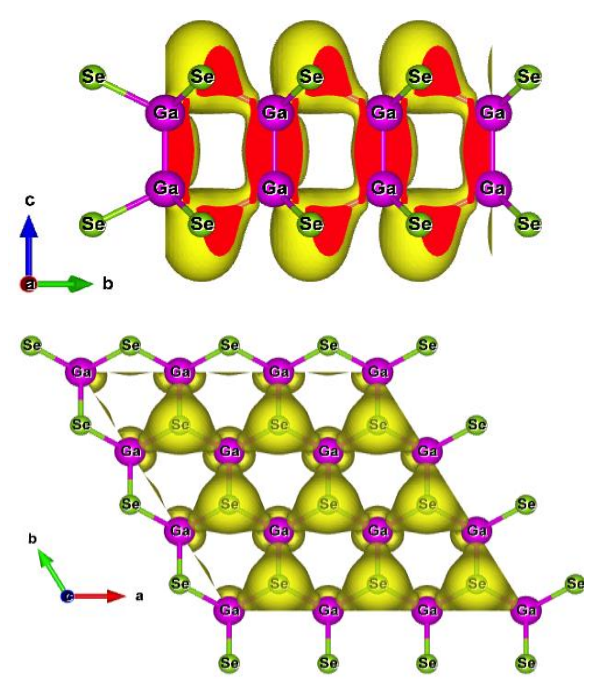

(b)

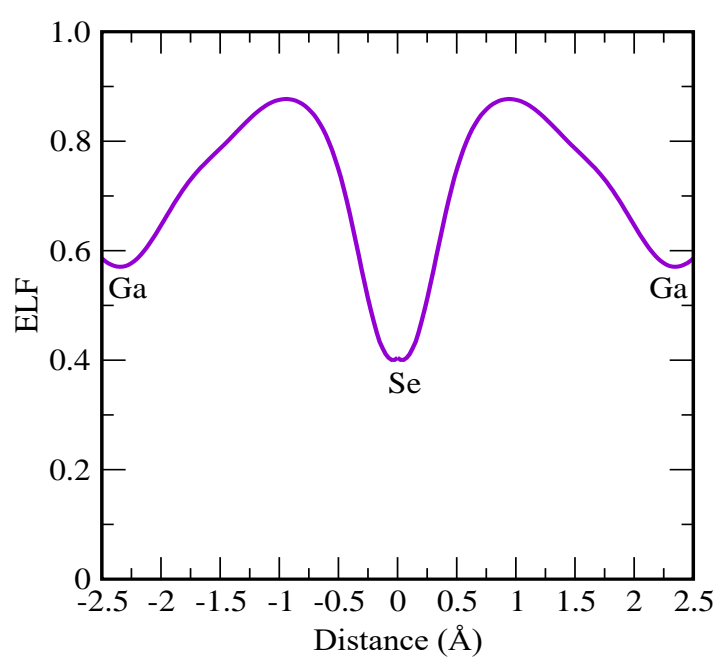

Figure 6 - (a) Localization domains of GaSe. The value of 0.66 chosen for the isosurface is convenient for visualization and corresponds to a significant degree of localization. (b) ELF profiles along the $\mathrm{Ga}-\mathrm{Se}-\mathrm{Ga}$ segments.

All these observations lead us to propose a possible correlation between the ionicity and the propensity of the $2 \mathrm{D}$ simulations to exhibit dynamical instability if the interlayer distance is not large enough.

Finally, we have calculated the electronic band structure of 2D-GaSe for two c parameters (24 $\AA$ and $27.6 \AA$, respectively) to investigate possible effect of the interlayer interaction on the electronic structure. For both values of the c-parameter used, this structure is very similar (Figure 7) and the $\varepsilon$-GaSe monolayer shows an indirect band gap with a value about $1.82 \mathrm{eV}$. This point is also important to note because it shows that the interlayer distance should be carefully considered during the simulations of the phononic properties but has little effect on the computation of electronic properties. 


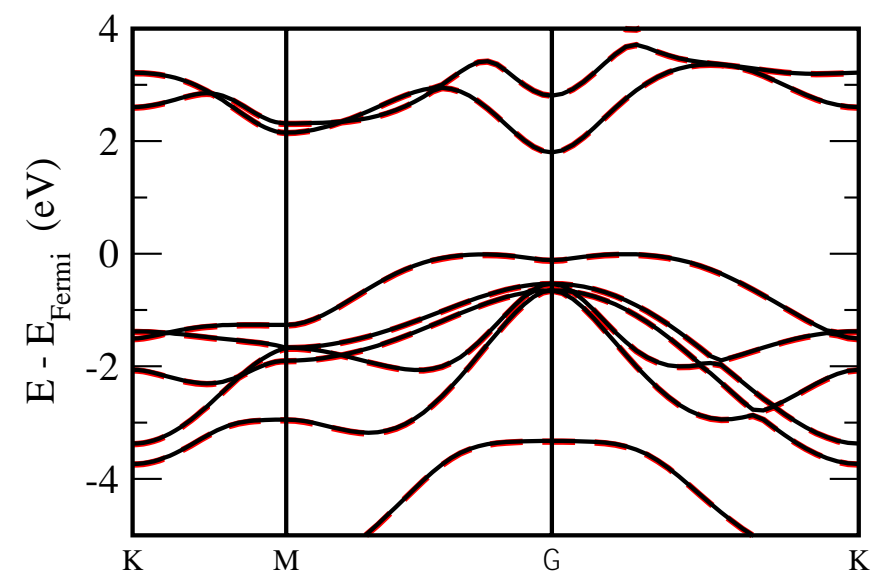

Figure 7 - Comparison of the calculated band structure for GaSe with a c-parameter of $24.3 \AA$ (red line) and 27.6 ̊ (black line)

\section{IV.Conclusions}

This work aims at tackling the issue related to the dynamical stability of simulated 2D materials. Thus, in some cases, phonon in a small pocket close the Brillouin zone center with imaginary frequencies appeared in simulations. The question we wanted to answer is the origin (physical or numerical) of this behavior and why it happens only in some compounds.

We used GaSe as a case study. We started by optimizing the 3D structure including van der Waal interactions and the results are in very good agreement with the experimental data. In particular, the phonon dispersion curves obtained by the small displacement method does not show any instability.

For the $2 \mathrm{D}$ configuration, the same formalism but without van der Waals interactions has been conducted using a simulation cell with a c-parameter (perpendicular to layers) increased by $50 \%$ compared to the $3 \mathrm{D}$ case. This leads to a dynamical instability using the small displacement method. Using a different methodology based on the perturbation approach (DFPT), this dynamical instability is lifted.

With a further increase of the spacing between layers, the dynamical instability also disappears when using the small-displacement method. This result is major as it indicates that contrary to the usual claiming, this effect is not numerical or computational artifact but originates in interlayer interaction that has not been 
cancelled because of an insufficient spacing between layers. The use of the smalldisplacement method should be used with great care, requiring the use of a large value of the c-axis, especially in the case of materials where long-range interactions may be foreseen i.e., in compounds with a marked ionic character as recent results in the epitaxy science proposed a link between the existence of such interactions and the ionicity of the materials.

\section{Acknowledgments}

S.R. acknowledges the support from MINECO, Spain Project No. MAT2016-75586C4-3-P. This work was supported by the LABEX iMUST (ANR-10-LABX-0064) of Universite de Lyon, France within the program "Investissements d'Avenir" (ANR-11IDEX-0007) operated by the French National Research Agency, France.

\section{References}

1 A. K. Geim, I. V. Grigorieva, Nature 499, 419 (2013).

2 A. Gupta, T. Sakthivel, S. Seal, Prog. Mat. Science 73, 44 (2015).

3 M. Xu, T. Liang, M. Shi, H. Chen, Chem. Rev. 113, 3766 (2013).

4 A. Splendiani , L. Sun , Y. Zhang, T. Li , J. Kim , C.-Y. Chim , G. Galli , F. Wang, Nano Lett. 10, 1271 (2010).

5 K. F. Mak , C. Lee , J. Hone , J. Shan , T. F. Heinz , Phys. Rev. Lett. 105, 136805 (2010).

6 D. C. Wallace, Thermodynamics of Crystals (J. Wiley, New York, 1972), pp. 32-41.

7 C. Ataca, H. Şahin, S. Ciraci, J. Phys. Chem. C 116, 8983 (2012).

8 H. Zheng, X.-B. Li, N.-K. Chen, S.-Y. Xie, W. Q. Tian, Y. Chen, H. Xia, S. B. Zhang, H.-B. Sun, Phys. Rev. B 92, 115307 (2015).

9 V. Zolyomi, N. D. Drummond, V. I. Fal'ko, Phys. Rev. B 89, 205416 (2014).

10 L. Debbichi, H. Kim, T. Bjorkman, O. Eriksson, S. Lebegue, Phys. Rev. B 93, 245307 (2016).

11 D. J. Late, B. Liu, J. Luo, A. Yan, H. S. S. Ramakrishna Matte, M. Grayson, C. N. R. Rao, V. P. Dravid, Adv. Mater. 24, 3549 (2012). 
12 V. Zolyomi, N. D. Drummond, V. I. Fal'ko, Phys. Rev. B 87195403 (2013).

13 X. Li, M.-W. Lin, A. A. Puretzky, J. C. Idrobo, C. Ma, M. Chi, M. Yoon, C. M.

Rouleau, I. I. Kravchenko, D. B. Geohegan, K. Xiao, Scientif. Rep. 4, 5497 (2014).

14 S. Lei, L. Ge, Z. Liu, S. Najmaei, G. Shi, G. You, J. Lou, R. Vajtai, P. M. Ajayan, Nano Lett. 13, 2777 (2013).

15 Y. Ma, Y. Dai, M. Guo, L. Yu, B. Huang, Phys. Chem. Chem. Phys. 15, 7098 (2013).

16 L. Huang, Z. Chen, J. Li, RSC Adv.5, 5788 (2015).

17 L. Ao, H. Y. Xiao, X. Xiang, S. Li, K. Z. Liu, H. Huang, X. T. Zu, Phys. Chem. Chem. Phys. 17, 10737 (2015).

18 A. Kuhn, A. Chevy, and R. Chevalier, Phys. Status Solidi A 31, 469 (1975).

19 P. Hu, Z. Wen, L. Wang, P. Tan, K. Xiao, ACS Nano 6, 5988 (2012).

20 G. Kresse, J. Furthmüller, Computational Materials Science. 6, 15 (1996).

21 G. Kresse, J. Furthmüller, Phys. Rev. B. 54, 11169 (1996).

22 J.P. Perdew, K. Burke, M. Ernzerhof, Phys. Rev. Lett. 77, 3865 (1996).

23 S. Grimme, J. Antony, S. Ehrlich, H. Krieg, J. Chem. Phys. 132, 154104 (2010).

24 D. Alfè, Comput Phys Commun. 180, 2622 (2009).

25 S. Baroni, S. de Gironcoli, A. Dal Corso, P. Giannozzi, Rev Mod Phys. 73, 515 (2001).

26 A. Togo, I. Tanaka, Scr Mater. 108, 1 (2015).

27 See Supplemental Material at [URL will be inserted by publisher] for the calculated PV curves for PBE and PBEvdW, and a comparison of the 3D structures obtained for PBE and PBEvdW for a similar value of the c-parameter.

28 A. Kuhn, A. Chevy, R. Chevalier, Acta Crystallogr., Sect. B: Struct. Crystallogr. Cryst. Chem., 32, 983 (1976).

29 K.R. Allakhverdiev, E.Yu. Salaev, M.M. Tagyev, S.S. Babaev, Solid State Conm. 59, 133 (1986).

30 K.-A.N. Duerloo, Y. Li, E.J. Reed, Nature Commun. 5, 4214 (2014).

31 Y.-C. Lin, D. O. Dumcenco, Y.-S. Huang, K. Suenaga, Nature Nanotech. 9, 391 (2014). 
32 K.-A.N. Duerloo, E.J. Reed, ACS Nano 10, 289 (2016).

33 D. Akinwande, C.J. Brennan, J. S. Bunch, P. Egberts, J.R. Felts, H. Gao, R. Huang, J.-S. Kim, T. Li, Y. Li, K. M. Liechti, N. Lu, H.S. Park, E.J. Reed, P. Wang, B.I. Yakobson, T. Zhang, Y.-W. Zhang, Y. Zhou, Y. Zhu, Extreme Mechanics Letters 13, 42 (2017)

34 T.J. Wieting and J.L. Verble, Phys. Rev. B 5, 1473 (1972).

35 Y. Kim, S. S. Cruz, K. Lee, B. O. Alawode, C. Choi, Y. Song, J. M. Johnson, C.

Heidelberger, W. Kong, S. Choi, K. Qiao, I. Almansouri, E. A. Fitzgerald, J. Kong, A. M. Kolpak, J. Hwang, J. Kim, Nature 544, 340 (2017).

36 W. Kong, H. Li, K. Qiao, Y. Kim, K. Lee, Y. Nie, D. Lee, T. Osadchy, R.J. Molnar, D. K. Gaskill, R. L. Myers-Ward, K. M. Daniels, Y. Zhang, S. Sundram, Y. Yu, S. Bae, S. Rajan, Y. Shao-Horn, K. Cho, A. Ougazzaden, J. C. Grossman, J. Kim, Nat. Mater. 17, 999 (2018).

37 N. Kuroda and Y. Nishina, J. Phys. Soc. Jap. 50, 2969 (1981).

38 T. Tanabe, S. Zhao, Y. Sato, and Y. Oyama, J. Appl. Phys. 122, 165105 (2017).

39 L. Pauling, The Nature of the Chemical Bond (3rd edn) (New York: Cornell University Press, 1960).

40 M.B. Hannay and C.P. Smyth J. Am. Chem. Soc. 68, 171 (1946) 